\title{
Bisphosphonates in bone cement inhibit PMMA particle induced bone resorption
}

\author{
Afsaneh Sabokbar, Yosuke Fujikawa, David W Murray, Nicholas A Athanasou
}

\begin{abstract}
Objective-Wear particle induced bone resorption is thought to be one of the mechanisms that contribute to implant loosening. It has previously been shown that macrophages, in response to polymethylmethacrylate (PMMA) particles, differentiate into bone resorbing osteoclasts, and that this process is inhibited by a bisphosphonate, etidronate (EHDP). The aim of this study was to determine whether incorporating EHDP in bone cement could reduce PMMA associated bone resorption.

Methods-Two concentrations of EHDP were mixed with PMMA monomer before polymerisation. Particles of PMMA (1-10 $\mu \mathrm{m})$ were generated then added to mouse monocytes cocultured with UMR106 rat osteoblast-like cells and the extent of osteoclast differentiation was determined by assessing the extent of tartrate resistant acid phosphatase (TRAP) staining and measuring the amount of lacunar bone resorption.

Results-The addition of PMMA to
\end{abstract} monocyte-UMR106 cocultures resulted in a marked increase in the number of TRAP positive osteoclast-like cells and a significant increase in the number of lacunar resorption pits compared with control cultures to which no particles had been added. After the addition of particles of PMMA + $20 \mathrm{mg}$ EHDP, significantly fewer lacunar pits $(p=0.00006)$ and fewer TRAP positive cells were noted compared with cocultures containing PMMA particles alone.

Conclusions-These results indicate that by mixing a bisphosphonate with bone cement, it is possible to inhibit PMMA particle induced bone resorption. This bisphosphonate inhibition of PMMA biomaterial wear particle containing macrophage-osteoclast differentiation and bone resorption may provide a possible therapeutic strategy to prevent or to control the osteolysis of aseptic loosening.

(Ann Rheum Dis 1998;57:614-618)

Nuffield Orthopaedic Centre, University of Oxford, Oxford

Correspondence to: Dr N A Athanasou, Department of Pathology, Nuffield Orthopaedic Centre, Headington, Oxford, OX3 7LD.

Accepted for publication 29 July 1998 implant components. ${ }^{1}$ The extent of this mor nuclear phagocyte response correlates with the amount of wear debris produced, and this in
Aseptic loosening of implant components is a ajor complication of cemented total macrophage polykaryons are commonly found in response to polymethylmethacrylate (PMMA) and other implant derived wear particles in the fibrous membrane surrounding loose turn may be dependent upon the stability of the implant. ${ }^{2}$ Numerous wear particles and a heavy macrophage infiltrate have often been reported in cases of early loosening of cemented implants whereas, in contrast, in the membrane surrounding a well fixed cemented prosthesis, little or no macrophage response is seen. ${ }^{3-5}$

The heavy foreign body macrophage response to PMMA bone cement and other biomaterial wear particles is thought to contribute to the osteolysis of aseptic loosening either by release of wear particle induced factors that stimulate bone resorption such as prostaglandins, interleukin 1 (IL1), IL6, and tumour necrosis factor $\alpha(\mathrm{TNF} \alpha),{ }^{6-8}$ or by providing a large population of immature mononuclear phagocyte precursor cells for differentiation into mature bone resorbing osteoclasts. ${ }^{9}$ We have recently shown that mouse and human macrophages responding to PMMA and other implant biomaterial particles, under specific cellular and hormonal conditions, are able in vitro to differentiate into mature osteoclasts capable of resorbing bone. ${ }^{9-11}$ The specific culture conditions required for this to occur are the presence of osteoblastic cells and 1,25 dihydroxy vitamin $\mathrm{D}_{3}\left(1,25(\mathrm{OH})_{2} \mathrm{D}_{3}\right)$, both of which would be present in the microenvironment of the bone implant membrane interface. Osteoblasts line the bone surface and inflammatory macrophages are known to secrete $1,25(\mathrm{OH})_{2} \mathrm{D}_{3} \cdot{ }^{12}$ We have also shown that this process can be inhibited by cytokines such as IL4 and leukaemia inhibitory factor $(\mathrm{LIF})^{13}$ as well as pharmacological inhibitors of bone resorption such as calcitonin and bisphosphonates. ${ }^{14} 15$

Bisphosphonates are synthetic compounds that inhibit bone resorption. They are commonly used to control the excessive osteolysis seen in osteoporosis, Paget's disease, and skeletal carcinomatosis. ${ }^{16}$ Bisphosphonates are known to influence osteoclastic bone resorption by a number of means including direct or indirect suppression of osteoclast function, inhibition of osteoclast differentiation, and promotion of osteoclast apoptosis. ${ }^{17-19}$ We have recently shown that the bisphosphonate compound, disodium ethane-1-hydroxy-1, 1-diphosphonate (etidronate (EHDP)) also known as 1-hydroxyethylidene-1, 1-bisphosphonic acid, can inhibit PMMA induced osteoclast generation and bone resorption. ${ }^{15}$ In this study we have sought to determine whether mixing bisphosphonates with PMMA bone cement can inhibit wear debris induced osteolysis and provide a possible therapeutic approach to prevent aseptic loosening. 


\section{Methods}

PMMA PARTICLES

PMMA with $9.2 \% \mathrm{BaSO}_{4}$ was provided by CMW (Depuy, UK). Tablets of EHDP were purchased from Procter and Gamble (UK) and were crushed to a powder form. They were then evenly mixed with PMMA monomer at final concentrations of $5 \mathrm{mg}$ and $20 \mathrm{mg}$ per gram of monomer. Both PMMA cement with no additives and PMMA containing EHDP, were then polymerised according to the manufacturer's instructions. Particles were produced from the polymerised cement by AEA Technology plc (Harwell, UK), the bone cement being crushed using a steel mortar pestle and then a Tema Micromill (Siebtechnik, Germany). Size distribution was determined by scanning electron microscopy. This demonstrated the majority $(>80 \%)$ of the particles were between 1 and 10 microns. The particles were then weighed, suspended in culture medium, and sonicated for 10 minutes before being added to culture wells at a concentration of $50 \mu \mathrm{g}$ per $\mathrm{ml}$, which we have previously found to be optimal. ${ }^{11}$ The particle suspensions were regularly tested for endotoxin activity (E-Toxate, Sigma, UK) and discarded if the test results were positive.

CULTURE CONDITIONS

For cell culture, alpha minimal essential medium (MEM) (Gibco, UK) was supplemented with $100 \mathrm{IU} / \mathrm{ml}$ penicillin, $10 \mu \mathrm{g} / \mathrm{ml}$ streptomycin and $10 \mathrm{mM}$ l-glutamine (Gibco, UK), and 10\% fetal calf serum (FCS) (Gibco, UK) (MEM/FCS). The cloned hormone responsive osteoblast-like UMR106 rat cell line, originally derived from a rat osteosarcoma, was generously provided by Professor T J Martin (Melbourne, Australia). These UMR106 cells have been shown to be tartrate resistant acid phosphatase (TRAP) negative, calcitonin receptor negative and are not capable of bone resorption. ${ }^{14}$ All incubations were carried out at $37^{\circ} \mathrm{C}$ in $5 \%$ carbon dioxide.

ISOLATION OF MURINE MONOCYTES

Circulating monocytes were isolated from the peripheral blood of female MF1 mice. Whole blood was diluted 1:4 in MEM and the monocytes were separated from whole blood by Ficoll-Hypaque (Pharmacia, UK) gradient centrifugation $(693 \mathrm{~g})$ for 20 minutes. The mononuclear cells in the interface layer were resuspended in MEM/FCS and the number of viable cells was determined using a haemocytometer.

PREPARATION OF COCULTURES ON HUMAN BONE SLICES AND COVERSLIPS

Monocyte cultures were set up on sterile $6 \mathrm{~mm}$ glass coverslips and human femoral cortical bone slices $\left(10 \mathrm{~mm}^{2}\right)$ as previously described. ${ }^{90}$ Bone slices and glass coverslips were placed in $7 \mathrm{~mm}$ wells of a 96 well tissue culture plate (Nunc, UK) before the addition of UMR-106 cells at a final concentration of 1 $\times 10^{4}$ cells per well. After 24 hours incubation, the monocyte cell suspension was then added at a final concentration of $1 \times 10^{5}$ cells per well. Control monocyte cultures were also set up in the absence of UMR 106 cells.
After two hours incubation, the bone slices and coverslips were removed from the 96 well plates, washed vigorously in MEM to remove non-adherent cells, and then transferred to 17 mm wells containing $1 \mathrm{ml} \mathrm{MEM/FCS}$ with $1 \times$ $10^{-7} \quad M \quad 1,25$ dihydroxy vitamin $\mathrm{D}_{3}$ $\left(1,25(\mathrm{OH})_{2} \mathrm{D}_{3}\right.$; Solvay Duphar, the Netherlands) and $1 \times 10^{-8} \mathrm{M}$ hydrocortisone (Sigma Chemicals, UK). At this point $50 \mu \mathrm{g} / \mathrm{ml}$ of PMMA \pm EHDP particles were added to the wells. As controls, additional wells were also set up where no particles were added. The cultures were maintained for 1,7 , and 14 days, with the media containing $1,25(\mathrm{OH})_{2} \mathrm{D}_{3}$ and hydrocortisone being replenished every three days.

HISTOCHEMICAL AND IMMUNOHISTOCHEMICAL CHARACTERISATION OF CULTURED CELLS After one and seven days, coverslips were removed and histochemically stained for the osteoclast associated enzyme TRAP ${ }^{21}$ using a kit from Sigma Chemicals (UK). In addition, coverslips were stained immunohistochemically by an indirect immunoperoxidase method to determine the presence of F4/80 antigen, which is expressed by murine monocytes and macrophages but not osteoclasts. ${ }^{22}$ In control and EHDP treated cultures, two glass coverslips were assessed at both one and seven days for TRAP and F4/80 staining. Long term cultures on coverslips are assessed after seven days rather than 14 days as this facilitates the analysis of histochemical and immunohistochemical staining. ${ }^{14} 15$

EXAMINATION BY SCANNING ELECTRON MICROSCOPY (SEM) OF BONE SLICES TO DETERMINE RESORPTION PIT FORMATION After one and 14 days in culture, the bone slices were removed from culture wells and (a) washed in phosphate buffered saline, (b) treated with $0.25 \%$ trypsin, (c) rinsed vigorously in distilled water, and (d) immersed in $0.25 \mathrm{M} \mathrm{NH}_{4} \mathrm{OH}$ for 24 hours. After alcohol dehydration, the bone slices were mounted onto aluminium stubs (Abbot Laboratories, UK), sputter coated with gold, and examined using a Philips SEM 505 scanning electron microscope.

\section{ASSESSMENT OF BONE RESORPTION AND} STATISTICAL ANALYSIS

The results were expressed as the number of bone resorption pits per bone slice; this has been shown to provide a good correlation with the total surface area of bone resorbed as the volume and area of individual resorption pits is known to fall within a defined range. ${ }^{23}{ }^{24}$ In each experiment four bone slices were used for each individual treatment (that is, control, PMMA alone, PMMA + EHDP (5 mg), and PMMA + EHDP $(20 \mathrm{mg}))$ and the mean number of pits per slice was calculated. Each experiment was carried out five times. The data from the experiments were analysed using analysis of variance and two tailed paired Student's $t$ test $(\mathrm{n}=5)$. 

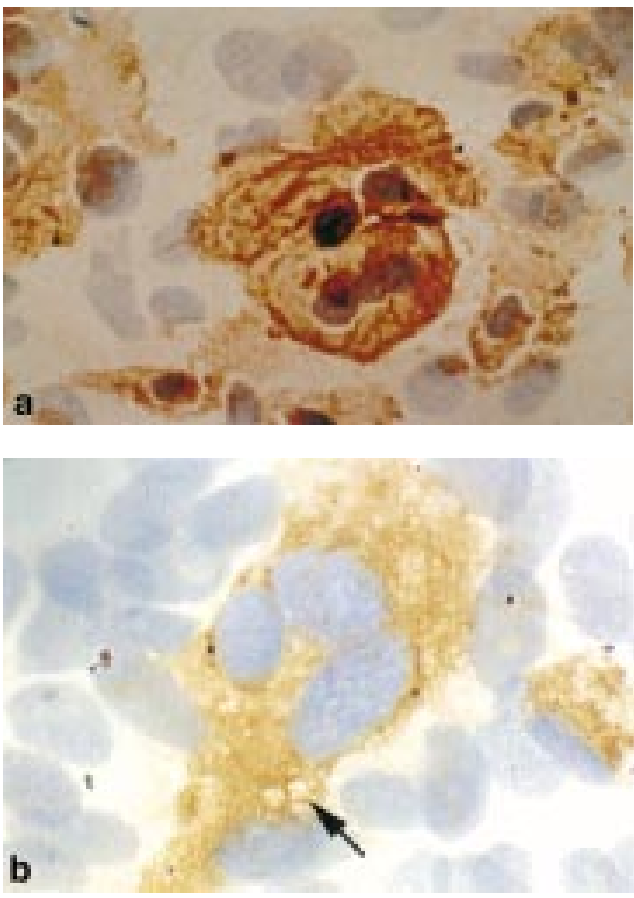

Figure 1 (a) Indirect immunoperoxidase staining for F4/80 antigen of 24 hour coculture of UMR106 cells and monocytes showing positive reaction (brown staining) for F4/80 antigen on isolated cells $(a)$ in the absence $(\times 630)$, and (b) in the presence of PMMA particles that have been phagocytosed (arrow) ( $\times 1000$, oil immersion).

\section{Results}

HISTOCHEMICAL AND IMMUNOHISTOCHEMICAL CHARACTERISATION OF ISOLATED CELLS

Adherent isolated cells, after 24 hours incubation, both in the presence or absence of UMR106 cells, were characterised as macrophages on the basis that they were strongly positive for F4/80 antigen (fig 1a), a monocyte/ macrophage marker that is not expressed by osteoclasts, and entirely negative for TRAP, an osteoclast marker. Cultures to which PMMA \pm EHDP particles had been added contained isolated adherent $\mathrm{F} 4 / 80$ positive cells that had phagocytosed PMMA particles (fig $1 \mathrm{~b}$ ) or were aggregated around larger fragments of PMMA.

After seven days incubation, both in the presence and absence of $1,25(\mathrm{OH})_{2} \mathrm{D}_{3}$ and UMR106 cells, monocyte derived macrophages positive for $\mathrm{F} 4 / 80$ antigen were noted in both test and control cultures. In cocultures of UMR106 cells and monocyte derived macrophages to which no particles had been added, a few TRAP positive clusters were seen after seven days incubation. In seven day cocultures of UMR106 cells and monocyte derived macrophages containing PMMA particles (with no EHDP), incubated in the presence of $1,25(\mathrm{OH})_{2} \mathrm{D}_{3}$, large numbers of TRAP positive cells including several large discrete clusters of TRAP positive cells were noted (fig $2 \mathrm{a}$ ). In contrast, the number of TRAP positive cells and clusters was absent or markedly reduced in seven day UMR 106-monocyte cocultures to which particles of PMMA + EHDP had been added (fig $2 \mathrm{~b}$ ). This reduction in the number of TRAP positive cells was more apparent in wells containing PMMA $+20 \mathrm{mg}$ of EHDP compared with PMMA + $5 \mathrm{mg}$ EHDP. The number
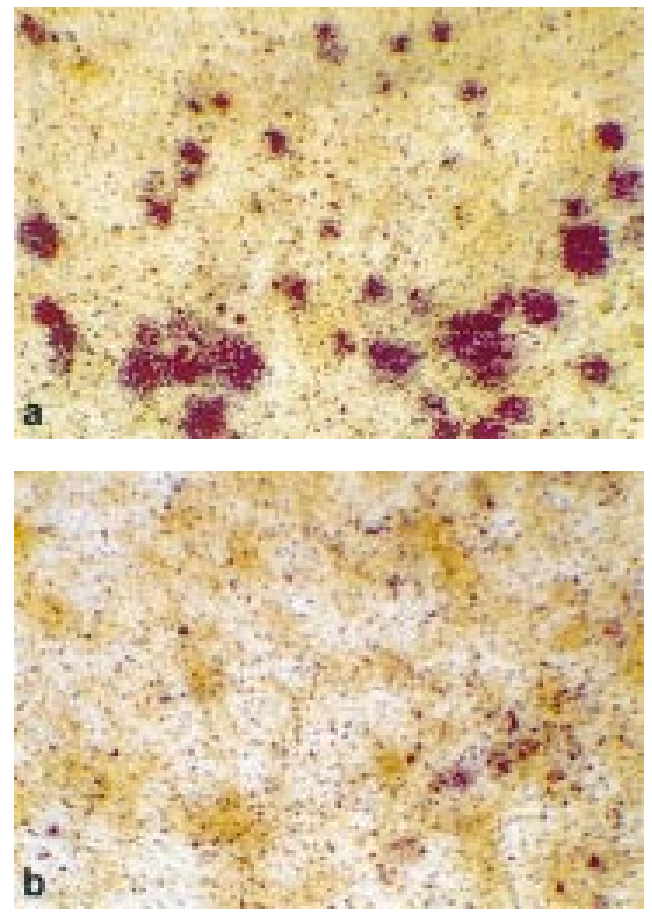

Figure 2 Histochemical staining for TRAP in seven day cocultures of UMR106 cells and murine monocytes showing: (a) numerous clusters of TRAP ${ }^{+}$cells in control cocultures containing PMMA particles $(\times 100)$; $(b)$ reduction of TRAP' cell cluster formation in cocultures containing PMMA particles $+20 \mathrm{mg} E H D P(\times 100)$.

of $\mathrm{F} 4 / 80$ positive cells in these cultures remained the same as control wells, thus indicating the lack of cytotoxic effects of EHDP.

In the absence of UMR106 cells or $1,25(\mathrm{OH})_{2} \mathrm{D}_{3}$, TRAP positive cell clusters were not seen in seven day control or PMMA containing monocyte cultures, indicating that osteoclast differentiation had not occurred.

ASSESSMENT OF BONE RESORPTION

After 24 hours incubation, there was no evidence of lacunar resorption on bone slices upon which monocytes had been cultured with UMR106 cells and $1,25(\mathrm{OH})_{2} \mathrm{D}_{3}$, both in the presence or absence of PMMA particles. However, after 14 days incubation, in the presence of $1,25(\mathrm{OH})_{2} \mathrm{D}_{3}$, numerous well defined resorption pits were noted in all UMR 106-monocyte cocultures, including those to which PMMA particles had been added

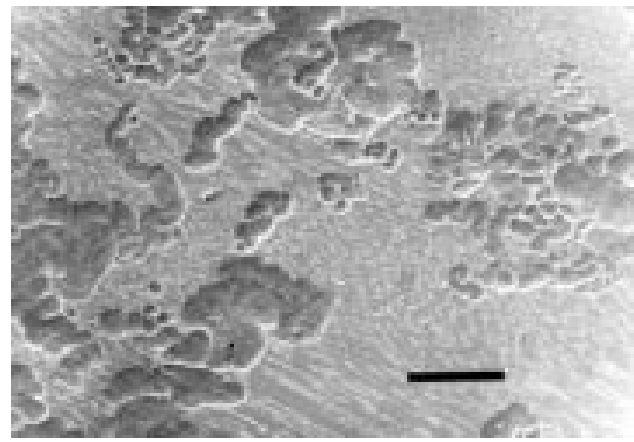

Figure 3 SEM photomicrograph of human bone slice cultured for 14 days in the presence of UMR106 cells, mouse monocytes, $1,25(\mathrm{OH})_{2} \mathrm{D}_{3}$ and PMMA particles. This shows extensive lacunar bone resorption with the formation of numerous resorption pits (black bar $=100 \mu \mathrm{m})$. 


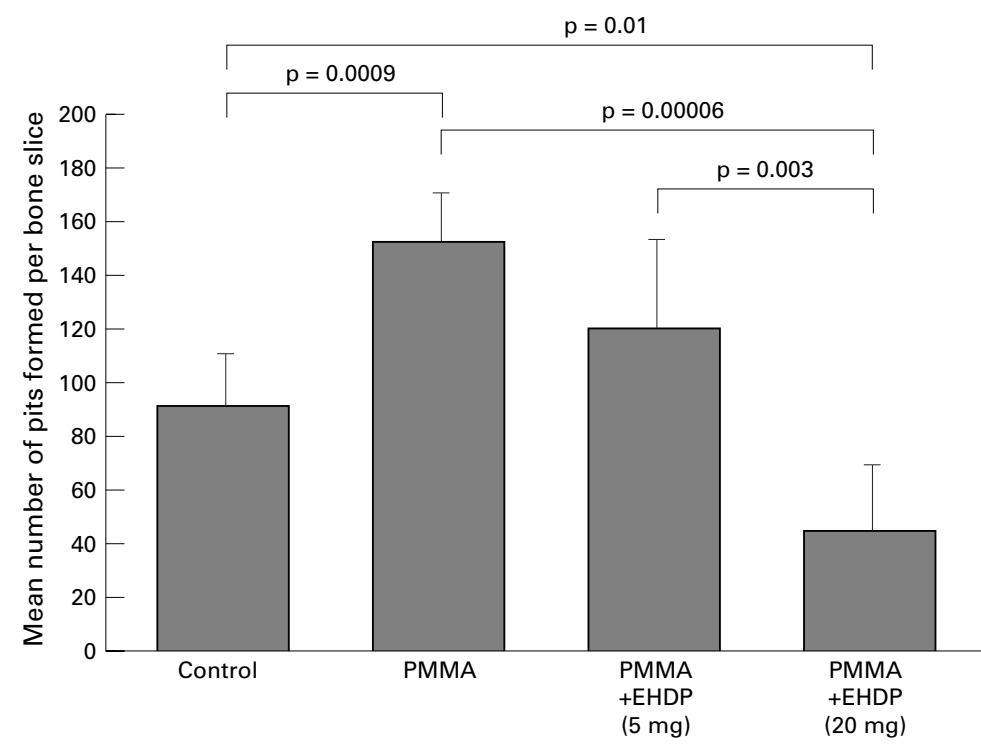

Figure 4 Effect of addition of particles of PMMA +EHDP (5 and $20 \mathrm{mg}$ ) and PMMA alone on the mean number of lacunar pits formed on bone slices after 14 days incubation in the presence of UMR106 cells and mouse monocytes. Levels of significance, using Student's paired t test, are indicated. that mononuclear phagocytes present in the inflammatory foreign body macrophage response to PMMA and other biomaterial wear particles are capable of differentiating into osteoclastic bone resorbing cells. ${ }^{910}$ In this study we have shown that when EHDP is mixed with particles of PMMA bone cement, there is significant inhibition of osteoclast differentiation by macrophages that have phagocytosed PMMA particles and that this is associated with a significant decrease in the amount of osteoclast mediated lacunar bone resorption.

We have previously shown that mononuclear phagocyte osteoclast precursors are present in the granulomatous inflammatory foreign body macrophage infiltrate that forms in response to subcutaneously implanted PMMA wear particles, and that monocytes that have phagocytosed PMMA particles, under specific cellular and hormonal conditions, are capable of differentiating into osteoclastic cells capable of extensive lacunar bone resorption. ${ }^{9-11}{ }^{13}$ The addition of EHDP to cocultures of UMR106 cells and monocyte derived macrophages has been found to inhibit the extent of lacunar bone resorption by osteoclasts partly through an effect on macrophage-osteoclast differentiation. ${ }^{15}$ In this study we have shown that the addition of particles of PMMA containing EHDP to monocyte derived macrophages similarly inhibits PMMA associated macrophage-osteoclast differentiation and bone resorption and that this activity does not affect the viability of cultured cells. The extent of this resorption was significantly less than that seen in cocultures containing particles of PMMA alone and than that seen in control cocultures with no added particles, suggesting that incorporation of a bisphosphonate into bone cement to inhibit macrophage-osteoclast differentiation may effectively be used to control periprosthetic osteolysis.

Previous studies have shown that EHDP acts to inhibit the phase of proliferation and maturation of mononuclear phagocyte precursors in osteoclast differentiation. ${ }^{5}$ It is known that bisphosphonates can preferentially inhibit proliferation of macrophages in concentrations that do not affect the viability of non-proliferating cells. ${ }^{27}$ Using a mouse bone marrow/calvaria coculture system, where alendronate was shown to inhibit $1,25(\mathrm{OH})_{2} \mathrm{D}_{3}$ induced osteoclastogenesis, ${ }^{28}$ it was suggested that this inhibitory effect may result from inhibition of the fusion of mononuclear osteoclast precursors to form multinucleated cells. Our previous studies have shown that, in monocyte-UMR106 cocultures, adding EHDP at the commencement of the coculture period abolishes bone resorption, whereas adding it after seven days coculture results in little or no inhibition of TRAP expression or bone resorption. ${ }^{15}$

The possibility that bisphosphonates act through mechanisms other than macrophageosteoclast differentiation to inhibit bone resorption is not excluded by our findings. A direct effect on the bone resorbing activity of mature osteoclasts is well recognised. Bisphosphonates are known to change the morphology of osteoclasts on the bone surface ${ }^{29}$ and it has been shown that pretreating bone particles with stimulate bone resorption by influencing activity of bone cells (both osteoblasts and osteoclasts). ${ }^{6-8}$ In addition, it has been shown 
bisphosphonates suppresses macrophage mediated and osteoclastic bone resorption. ${ }^{30}{ }^{31}$ In rat calvarial cultures containing the macrophagelike cell line (J774), Horowitz et $a^{\beta 2}$ also showed that disodium pamidronate inhibits PMMA associated bone resorption as determined by a reduction in ${ }^{45} \mathrm{Ca}$ release. Another way by which bisphosphonates could directly influence osteoclast mediated bone resorption is by increasing osteoclast apoptosis, thus, shortening the life span of these cells. ${ }^{19} \mathrm{~A}$ further possible mode of action whereby bisphosphonates could act on osteoclast differentiation is via an effect on cells of the osteoblast lineage that are known to influence the recruitment and differentiation of mononuclear phagocyte osteoclast precursors. ${ }^{33}$ Bisphosphonates have been shown to inhibit the osteoblast mediated production of IL6, ${ }^{34}$ a cytokine that is known to play a part in osteoclast formation. ${ }^{35}$ Recent in vitro work has also suggested that bisphosphonates induce the synthesis and release of a fragment of PTH related peptide by osteoblastic cells, which may then directly inhibit osteoclastic bone resorption. ${ }^{36}$

In the same way that antibiotics have been mixed with PMMA and used effectively to control infection, it is possible that mixing a pharmacological inhibitor of bone resorption such as a bisphosphonate with bone cement may be used to prevent or to control the bone resorption seen in aseptic loosening. The initial release of antibiotics from bone cement tends to be at high concentrations; this is followed by gradual diffusion of antibiotic at lower concentrations into the surrounding tissue.$^{37}$ If bisphosphonates were to be included in bone cement one would similarly expect that an initial high dose release of bisphosphonates, which would then decrease to a lower dose over time. Roentgen stereophotogrammetric analysis studies have demonstrated that implants subside into the bone in the early postoperative period and that those that migrate more rapidly are more likely to fail early. This early migration is thought to occur as a result of osteolysis. ${ }^{38}$ If bisphosphonates were to be incorporated in the cement the initial release of these compounds at high concentration may decrease this early osteolysis, minimise migration, and consequently, reduce the risk of implant loosening.

This study was supported by a grant from the Wellcome Trust.

1 Lennox DW, Schofield BW, McDonald DF, Riles LH. A histologic comparison of aseptic loosening of cemented, press-fit and biologic ingrowth prostheses. Clin Orthop press-fit and biologi

2 Mjoberg B. Theories of wear and loosening in hip prostheses: wear-induced loosening vs. loosing induce wear - a review. Acta Orthop Scand 1994;65:361-71

3 Linder L, Carlsson AS. The bone-cement interface in hip arthroplasty: a histologic and enzyme study of stable components. Acta Orthop Scand 1986;57:495-500.

4 Malcolm AJ. Pathology of long-standing cemented total hip replacement in Charnley's cases. J Bone Joint Surg 1988;70B(suppl): 153

5 Maloney WJ, Jasty M, Burke DW, O’Connor DO, Zalenski $\mathrm{EB}$, Brydon $\mathrm{C}$, et al. Biomechanical and histologic investigation of cemented total hip arthroplasties: a study of autopsy-retrieved femurs after in vivo cycling. Clin Orthop 1989;249:129-40.

6 Murray DW, Rushton N. Macrophages stimulate bone resorption when they phagocytose particles. J Bone Joint resorption when they phag
Surg 1990;72B:988-92.

7 Jiranek WA, Machado M, Jasty M, Jervevar D, Wolfe HJ, Goldring SR, et al. Production of cytokines around Goldring SR, et al. Production of cytokines around Surg 1993;75A:863-79.
8 Kim KJ, Rubash HE, Wilson SC, D'Antonio JA, McClain EJ. A histologic and biochemical comparison of the EJ. A histologic and biochemical comparison of the interface tissues in cementless and
ses. Clin Orthop 1993;287:142-52.

9 Quinn JM, Joyner C, Triffitt JT, Athanasou NA. PMMAinduced inflammatory macrophages resorb bone. J Bone Joint Surg 1992:74B:652-8.

10 Pandey R, Quinn J, Joyner C, Murray DW, Triffitt JT, Athanasou NA. Arthroplasty implant biomaterial-associated macrophages differentiate into lacunar bone-resorbing cells. Ann Rheum Dis 1996;55:388-95.

11 Sabokbar A, Fujikawa Y, Murray DW, Athanasou NA. Radio-opaque agents in bone cement enhance bone resorption. J Bone Joint Surg 1997;79B:129-34.

12 Nathan CF. Secretory products of macrophages. J Clin Invest 1987;79:319-26.

13 Sabokbar A, Fujikawa Y, Brett J, Murray DW, Athanasou NA. Increased osteoclastic differentiation by polymethylmethacrylate wear particle-associated macrophages: inhibition by interleukin- 4 and leukaemia inhibitory factor. Acta tion by interleukin-4 and leuka

14 Quinn JMW, McGee JO'D, Athanasou NA. Cellular and hormonal factors influencing monocyte differentiation to osteoclastic bone resorbing cells. Endocrinology 1994;134: 2416-23.

15 Pandey R, Quinn J, Sabokbar A, Athanasou NA. Bisphosphonate inhibition of bone resorption by particulate
biomaterial-associated macrophages. Acta Orthop Scand 1996;67:221-8

16 Fleisch H. Bisphosphonates: Mechanisms of action and clinical applications. J Bone Miner Res 1983;1:319-57.

17 Carano A, Tietbelbaum SL, Kousek JD, Schlessinger PH, Blair HC. Bisphosphonates directly inhibit bone resorption activity of isolated avian osteoclasts in vitro. J Clin Invest 1990;85:456-61.

18 Sahni M, Guenther HL, Fleisch H, Collin P, Martin TJ. Bisphosphonates act on rat bone resorption through the mediation of osteoblasts. J Clin Invest 1993;91:2004-11.

19 Hughes DE, Wright KR, Uy HL, Sasaki A, Yoneda T, Roodman GD, et al. Bisphosphonates promote apoptosis in murine osteoclasts in vitro and in vivo. J Bone Miner Res 1995;10:1478-87.

20 Udagawa N, Takahashi N, Akatsu T, Tanaka H, Sasaki T, Nishihara T, et al. Origin of osteoclasts: Mature monocytes and macrophages are capable of differentiating into osteoclasts under a suitable microenvironment prepared by bone marrow-derived stromal cells. Proc Natl Acad Sci USA 1990;87:7260-64.

21 Minkin C. Bone acid phosphatase: tartrate-resistant acid phosphatase as a marker of osteoclast function. Calcif Tissue Int 1982;34:285-90.

22 Austyn JM, Gordon S. F4/80 a monoclonal antibody directed specifically against mouse macrophage. Eur J directed specifically again

23 Athanasou NA, Gray A, Revell PA, Fuller K, Cochrane T, Chambers TJ. Stereophotogrammetric observations on bone resorption by isolated osteoclasts. Micron and Microscopica Acta 1984;15:47-53.

24 Chambers TJ, Revell PA, Fuller K, Athanasou NA. Resorption of bone by isolated rabbit osteoclasts. J Cell Sci 1984; 66:383-99.

25 Revell PA. Tissue reactions to joint prostheses and the products of wear and corrosion. In: Berry C, eds. Current trends in pathology. Berlin: Springer-Verlag, 1982:73-101.

26 Bullough PG, DiCarlo EF, Hansraj KK, Neves MC. Pathologic studies of total joint replacement. Orthop Clin North Am 1988;19:611-25.

27 Cecchini MG, Fleisch H. Bisphosphonates in vitro specifically inhibit among the hematopoietic series, the development of the mouse mononuclear phagocyte lineage. J Bone ment of the mouse mononuc

28 Schmidt A, Rutledge SJ, Endo N, Opas EE, Tanaka H, Wesolowski G, et al. Protein tyrosine phosphatase activity regulates osteoclast formation and function: inhibition by alendronate. Proc Natl Acad Sci USA 1996;93:3068-73.

29 Sato M, Grasser W. Effects of bisphosphonates on isolated rat osteoclasts as examined by reflected light microscopy. J Bone Miner Res 1990;5:35-40.

30 Chambers TJ. Diphosphonates inhibit bone resorption in macrophages in vitro. J Pathol 1980;132:255-62.

31 Flanagan AM, Chambers TJ. Inhibition of bone resorption by bisphosphonates: interaction between bisphosphonates, osteoclasts and bone. Calcif Tiss Int 1991;49:407-15.

32 Horowitz SM, Algan SA, Purdon MA. Pharmacological inhibition of particulate-induced bone resorption. J Biomed Mater Res 1996;31:91-6.

33 Rodan GA, Fleisch HA. Bisphosphonates: mechanisms of Rodan GA, Fleisch HA. Bisphosphon
action. J Clin Invest 1996;97:2692-6.

34 Passeri G, Girasole G, Ulietti V, Giuliani N, Pedrazzoni M, Sartoni I, et al. Bisphosphonates inhibit IL-6 production by human osteoblastic cells MG-63. J Bone Miner Res 1994; 9:S230

35 Athanasou NA. The cellular biology of bone resorbing cells. J Bone Joint Surg 1996;78A:1096-112.

36 Vitte C, Fleisch H, Guenther HL. Bisphosphonates induce osteoblasts to secrete an inhibitor of osteoclast-mediated resorption. Endocrinology 1996;137:2324-33.

37 Wahlig H. Antibiotic-loaded acrylic cements: elution properties. In: Elsan RA, ed. Revision arthroplasty. Vol 2. London: Franklin Scientific Publications, 1983:56-63.

38 Alfaro J, Murray DW, O’Neill MA, Gunther T, Bulstrode C. Cement migration after THA. A comparison of Charnley and Exeter femoral stems with RSA. J Bone Joint Surg Br (in press). 